13 pages, from Dr. T. Thomson's “ System of Chemistry," adopting his atomic numbers, without the slightest allusion to the name of that chemist; but, instead of it, supplying that of his brother compiler, Phillips, who scarce ever determined an atomic weight in his life. We consider it necessary thus to caution our readers, although, perhaps, we might have spared ourselves the trouble, as we do not consider they can have any propensity to be dictated to by W.T. Brande, of her Majesty's Mint.

Elements of Natural Philosophy; being an Experimental Introduction to the Study of the Physical Sciences. By G. BIRD, M.D. 1829.

¿ Vous en savez l'auteur.

Non; mais je sais fort bien Qu'a ne le point flatter, son sonnet ne vaut rien."

THIS is a hasty production, which might be improved by copious and careful correction; but we doubt whether the author is quali. fied for the task, as he, obviously, is nurrowminded aud illiberal. Here is his dedication, pregnant with the grossest error- "To the Court of Examiners of the Society of Apothecaries of London, this work is respectfully dedicated, in humble, but sincere, acknowledgment of the benefits conferred upon the Medical Profession, by the improvements they have effected in the edu. cation of the practitioner of medicine, and the consequent higher position which he occupies in society." This is neither more nor less than a direct insult to the general practitioners of England, who have, by their own confession and public appeals, declared that they have been betrayed and enslaved by these very men, whom the writer of this book, from some selfish motive, dares thus to flatter.

\section{TREATMENT OF SCARLATINA.}

To the Editor of The Lancet.

Sxr:-As many valuable lives are (and especially of late have been) lost, from the fatal effects of scarlatina and other eruptive diseases, I hope you will afford to the accompanying remarks a place in the columns of THE LANCET:-
On Saturday, November 16th, an athletic young man, about 21 years of age, of robust constitution and sanguine temperament, after suffering for several days from severe dyspeptic symptoms, heartburn, nausea, and shiverings, was compelled to keep his room from the accession of sore throat, attended with the usual eruption of scarlet fever, much tumefaction of the neck and face, flushed countenance, obstinate vomiting and diarrhoea, the latter symptoms apparently the result of some irregularity of diet, \&c., for some days previous to the appearance of the dyspepsia. As the patient was plethoric, and the pulse strong and quick, trelve ounces of blood were taken from the arm, and twelve leeches applied to the throat, ten grains of the compound colocynth pill at once; an effervescing saline dranght every five hours.

Nov. 17. Sickness, vomiting, and diarrhoea; continue an astringent draught with a few drops of laudanum, given every five hours ; anodyne draught at bed-time.

18. An excessive quantity of mucus about the fauces appearing to be the cause of the continuation of the sickness: 12 grains of pulv. ipecac. were given to remove it; afterwards an aperient mixture, containing sulphate of magnesia, $\overline{3} j$; carb. of mag. $3 j ;$ water, $\zeta \mathrm{v}$; two large spoonsful every five hours. The first dose was rejected froin the stomach, but, in the afternoon, the vomiting began to abate, and, after taking one drop of hydrocyanic acid, in camphor mixture, it ceased. Extr. of aconite, gr. j; a pill at bed-time.

19. Passed a tranquil night, and slept composedly. The case being considered of an alarming character by the friends of the patient, two more medical men were called in consultation on this day, and I was an unwilling spectator rather than a cordial co-operator in the treatment which ensued. The patient having endured, for three days successively, extraordinary evacuations, by purging and vomiting; having been bled, generally and locally ; having, during these operations, been unable to take food, or to retain anything which he drank; being, also, in a state of great exhaustion, turning pale when raised up in bed, and unable to maintain the position beyond a very short time; the pulse having now become weak and rapid from irritability, arising from exhaustion and debility; I should have been contented to have exhibited, under these circumstances, mild sedatives at intervals, and small quantities of wine diluted in water. My view was to tranquillise the nervous system, and also to give a gentle stimulus to the circulation, in the severe struggle it had to maintain against the approach of typhus, or of malignant sore throat. These views were not acquiesced in by the other parties, but the treatment was nearly as follows:- 
Magnes. rhubarb, of each grs. $\mathrm{xx}$; water, zjjss. A draught at once and in the evening.

Solution of acet. of ammonia, 3 ss ; spir. of xitr. ather, $5 \mathbf{j}$; camphor mixiure, $\tilde{3}$ j. A draught every five hours.

In the course of the day the patient became excessively restless; stupor came on ; jactitation; delirium; speech unintelligible; fauces ulcerated; bowels purged.

20. Tongue coated over with a white fur; mouth clammy; stupor; stertorous breathing; pulse rapid.

Carb. of ammon., 3ij ; vater, 亏ैviii. Two spoonsful every five hours, with lime juice.

21. The same as yesterday.

22. Eight leeches applied in the course of the night to the temples; in the morning the patient had become decidedly typhoid; the mouth dry and brown; the pulse small and rery rapid; the strength exhausted; he could not raise himself in bed; can take no nourishment by the mouth.

23. Patient lies in a weak state, is delirious, and unable to swallow. Enemas of broth, yeast, and port wine, were now resorted to several times in a day; by dint of these, of a naturally stout constitution, and by great good fortune, in a few days he struggled through this fearful ordeal, the balance turned in his favour, and he slowly recovered. The question presents itself, might he not have been steered clear of such imminent danger? My opinion is, had the treatment, from the fourth day after the commencement, been of a soothing sedative, at the same time of a slightly stimulant cha. racter, instead of a directly lowering and weakening one, by the continuation of the purgatives, repetition of the bleeding, \& $c_{\text {, }}$, he would have avoided it. This patient's mother took the disease, but in a less vio. lent form; the dranghts of rhubarb and magnesia were given freely; she became very restless, weak, and a painful sensation of weight and constriction of the chest came on; she also took a saline effervescing mixture containing some vin. antim. She complained of excessive dryness and smarting of the mouth after each dose; the fauces were hot and dry, the tonsils ulcerated; she could not bear the painful feelings produced by the medicines, and resolutely eleclaring she would take no more of them; ordered for herself some sago with port wine in it, and a draught containing some chloride of morphia, which procured sleep; the painful constriction of the chest now entirely left her; she continued to take wine daily, and soun became iconvalescent. The case was protracted beyond due and natural limits by the too free use of purgatives, salines, antimonials, \&c., and she was confined to her room nearly three weeks.

A woman, about 60 years of age, a nurse to the above-named patients, caught the disease, which was characterised by the usual symptoms, rigors, succeeded by heat, pain in the forehead, redness, and tumefaction about the fauces, a sensation of rawness or soreness down the trachea to the bronchial tubes, troublesome cough, loss of appetite, sleepless nights. She went home to her cottage, and was entirely under my medical care.

As there was no particular reason to suppose any undue accumulation to exist in the bowels, they were not "scoured out," $s e$ cindum artem, but remedies applied to such immediate symptoms of importance as presented themselves; there were the cough, headach, sleeplessness, and irritability of pulse, or disturbed action of the heart. Extr. of aconite, extr. of hyos., ginger powder, each gr.j. A pill night and morning.

Tinct. of hyosc, 3 ss ; antim. vine, $5 \mathrm{j}$; sulph. of magnes., 3 ss.

Camphor mixture, 3 vijss. Two ounces every six hours.

The pills entirely removed the headach, cough, sleeplessness, and irritability of the circulation; the mix ture might be considered almost a "placebo"; however, by the time she had taken it, the bowels were gently acted on, not purged; she took half a dozen pills and two mixtures, every symptom of the disease vanished, she complained ouly of debility; she took a little wine twice aday, and her strength was soon restored; in the space of about five or six days from the commencement of the treatment, she returned to her post of nurse to the other sick persons of whom she had caught the disease, and who were still in bed, and in bad plight.

If an individual, thus treated, is reduced in strength, and thrown into a state of debility, requiring wine and strong nutriment for restoration, can we wonder, looking at the nature of the treatment by strong depletory measures, that this disease should so often run into the typhoid form of fever, or that phagedenic ulceration of the mouth or fauces should occur, so often fatal, and in all cases protracting the convalescence and cure?

A maid servant, aged about 20 years, in the same family, next caught the infection; her symptoms were, in all respects, more strongly developed than in the last case, she was entirely confided to my care; the remedies used were the same, with the exception of her taking a small dose of castor oil, as an aperient, instead of the mag. sulph.? she was never purged, the bowels were gently, but effectually, opened; the anodyne pills procured good nights, allayed the cough, and soothed the irritability of the circulation; the swelling of the tonsils and tumefaction of the fauces, subsided by resolution. In four or five days she felt no other ailment but debility; she was ordered to 
take a little wine twice a day; her appetite soon returned, and she resumed her station as nurse and waiting maid to the first patients, and to a third, whose case remains to be related. I have the strongest conviction, that in this ease, had there been practised the minia medicina diligentia, or, indeed, even but moderately depletory measures, or antiphlogistic treatment, as it is termed, the symptoms would probably have assumed the typhoid form, or phagedenic ulceration of the mouth or fauces have followed. This girl now became the nurse of another patient in the same house, a woman about fifty years of age, who, having officiated as nurse to some of the inmates, caught the infection; the disease commenced with all the symptoms similar to those in the cases just related. I prescribed for her as on the two former occasions; she began by taking the anodyne pills, they were followed by good effects, sleep at night, with composure of the circulation; the bowels were gently moved by a dose of castor oil ; the tumefaction of the fauces, which had appeared first on the right side, subsided, and the left became swollen: I had no fear but that in a day or two, that would, with equal facility, subside, by the continuation of the same means; I was disappointed in my expectation. One of the surgeons, who had been in attendance in consultation, called to see the famils, and, in my absence, prescribed for this patient, a strong dose of colocynth, calomel, and antimony, and an opening mixture of salts, castor oil, \&c.; a dose to be taken every five hours-he declaring that she wanted a good"scouring out."

This proceeding afforded me a fair opportunity of judging of the comparative merits of the two methods of treatment. On my visiting the patient the following morning, I found her bordering on the typhus fever, the tongue had become dry and coated, the eyes sunken, the pulse rapid and weak, the conntenance of a yellowish pale colour, and the fauces lad run into a state of ulcera. tion: she had suffered great pain in the bowels, had been purged excessively, had passed a sleepless and wretched night, and was in a condition infinitely worse than she was prior to this interference.

I watched her through the rest of her career, whicb was tedious and troublesome, occupying twice the time of the two lastnamed cases, and attended by anomalous symptoms, such as acute and lancinating pains in the limbs, moving from one to the other, and occasioning her great distress; a symptom from which those were wholly free who were treated cautiously, and with sedatives, \&c., but which afflicted, more or less, all the patients treated by active depletory means.

In conclusion, I beg to remark, that in scarlatina, measles, small-pox, and other eruptive fevers of a specific character, af. fecting the skin and mucous surfaces gene. rally, I have invariably observed that calo. mel, even in a small dose, is followed by a sudden extension of ulceration, if any existed about the fauces, or by the immediate conversion of the tumefaction of those parts into the state of ulceration. It is a matter of great and serious importance, that of distinguishing the effects of a "reo medy" ? from those the natural produets of the progress of disease. It is a mistake of the very worst kind, and most lamenta. ble in its consequences, not to distinguish accurately between the two. If a dose of calomel can so alter vital actions, that the progress of some diseases is facilitated and extended by it, how fatal must be its exhibition in such circumstances! That if does encourage the progress and ravages of scarlatina and other eruptive fevers, and hastens on their termination either in gan. grene or typhus, to which they have a natural tendency, I am fully convinced by long observation. It is not impossible for persons to recover who have taken calomel under such circumstances, but they do so with more difficulty and tediousness. Not only does calomel have this bad effect, but any very active depletory or antiphlogistic treatment, as we term it, will do the same thing, though not so suddenly or virulently.

The peculiar stupor of eruptive fevers I have found to yield best to the use of the mild class of sedatives before spoken of, which are, according to my experience, specific remedies in scarlatina, and diseases of the like nature, almost as certainlly as mercury in syphilis, or sulphur in psora. Gentle evacuants, and moderate local san. guineous depletion, may in some cases be proper and useful adjuvants. I remain, $\mathrm{Sir}_{;}$ Geo. Bodingrox.

Sutton Coldfield, Dec. 11, 1839.

\section{HYDATIDS OF THE MOUTH.}

\section{To the Editor of The LANCET.}

SIR:-By inserting the following case, if you consider it of sufficient importance, in your valuable Periodical, you will obligg your obedient servant,

M. D. Thompson, M.R.C.S.L.

Staleybridge, Jan. 7, 1840.

The diagnosis of hydatical diseases, when they occur in deep-seated parts, being generally so imperfectly understoud, that tho profession rarely contemplate the existerce of such diseases, when so situated, until they are either artificially or naturally es posed to the sight, I have been induced to forw ard you the following case:- 